\title{
Introdução à Mecânica dos "Quanta" Parte I*
}

\author{
Theodoro A. Ramos \\ Escola Politécnica de São Paulo
}

\begin{abstract}
Este artigo consiste nas notas da primeira conferência publicado originalmente no número de Novembro de 1931 no Boletim do Instituto de Engenharia, pp. 157-166. Aqui os conceitos básicos da física ondulatória e da óptica geométrica são discutidos e a analogia entre a mecânica clássica e a óptica geométrica é explorada com vistas à introdução da mecânica ondulatória de Schrödinger.
\end{abstract}

This paper consists of the first lecture notes published originally in the November 1931 issue of the Bulletin of the Engineering Institute, p. 157-166. Here the basic concepts of the physics of waves and geometrical optics are discussed and the analogy between the classical mechanics and geometrical optics is explored towards the introduction of the Schrödinger undulatory mechanics.

\section{Introdução}

Uma das principais tarefas da Física moderna tem sido a investigação e o estudo aprofundado das formas matemáticas abstratas nas quais se enquadrem satisfatoriamente os fenômenos físicos observados.

Não mais se hesita, atualmente, em abandonar as concepções "simplistas" de um mundo constituído por associações de partículas e de fluidos movendo-se no espaço de acordo com determinadas leis de força. Em sua evolução a Física teórica utiliza-se cada vez mais das formas abstratas; é possível que o progresso científico conduza a teorias físicas que não mais admitam as representações concretas sugeridas pela tradição clássica, e que se exprimam exclusivamente por intermédio de combinações de símbolos matemáticos.

No início do século passado prevalecia entre os homens de ciência a opinião de que a velha mecânica de Newton enfeixava os elementos necessários à explicação dos fenômenos físicos.

As idéias de Newton sobre a natureza corpuscular da luz eram ainda aceitas por sábios eminentes como Laplace e
Poisson.

A teoria ondulatória da luz foi a primeira teoria física que se desenvolveu apoiando-se em uma concepção radicalmente diferente da concepção newtoniana. A propagação da luz é aí assimilada à propagação de ondas. $\mathrm{O}$ fenômeno luminoso em um ponto caracteriza-se por uma função periódica do tempo denominada "variável luminosa". A intensidade da luz no ponto considerado é por definição o quadrado da amplitude da vibração luminosa. A variável luminosa deve também satisfazer à equação de propagação das ondas e a determinadas condições limites.

Quando a luz se propaga em um meio cujo índice de refração é constante, as superfícies de igual fase, em um dado instante, são planos paralelos cujas normais constituem os "raios" percorridos pela perturbação luminosa.

No caso de um meio refringente cujo índice é uma função de ponto lentamente variável as superfícies de igual fase adquirem curvatura e os raios deixam de ser retilíneos.

Em tais casos a intensidade luminosa ou é constante ou varia lentamente ao longo dos raios cuja forma é determinada pelo princípio de Fermat o qual afirma que "entre 2

${ }^{*}$ Com este artigo iniciamos a publicação da série de conferências sobre a Mecânica Quântica ministradas pelo Prof. Theodoro Ramos na Escola Politécnica do Rio de Janeiro em 1931. Quatro artigos foram publicados nos números de Novembro de 1931, Fevereiro de 1932 , Junho de 1932 e Agosto de 1933 do Boletim do Instituto de Engenharia. No nosso conhecimento, trata-se do primeiro texto didático sobre a Mecânica Quântica publicado no Brasil. No primeiro artigo, T. Ramos explora com fundamentação matemática e riqueza de detalhes a analogia entre os princípios da mecânica clássica e da óptica geométrica. A mecânica ondulatória é apresentada no segundo artigo seguindo os passos e analogias encontradas nas comunicações de Schrödinger que apareceram em 1926 no Annalen der Physik. A seguir a interpretação probabilística de Born é discutida em profundidade. No terceiro artigo, T. Ramos explora as origens básicas da mecânica quântica seguindo a formulação inicial de Heisenberg. Finalmente, em sua quarta conferência, a mecânica quântica matricial de Heisenberg, Born e Jordan é analisada concluindo com a demonstração da equivalência entre a mecânica ondulatória e a mecânica matricial. Com a publicação desta série, a RBEF procura resgatar trabalhos de eminentes professores que contribuíram para a introdução de teorias científicas "modernas" no Brasil ainda incipiente em pesquisa na área de Física. Por outro lado, o material pode contribuir eficientemente na aprendizagem dos conceitos e teorias fundamentais abordados nas disciplinas de História da Física e Mecânica Quântica dos cursos de Física. Para os interessados, recomendamos como leitura complementar o livro Quantum Mechanics de S. Tomonaga (North-Holland, 1968) e o artigo de R. Köberle, Sobre a Gênese da Mecânica Ondulatória, Rev. Bras. Fis. v. 9, n. 1 (1979). Agradecemos ao Prof. Ildeu de Castro Moreira por nos ter chamado atenção para este importante documento da história da física brasileira e ao Prof. Salomon Mizrahi pela leitura do texto editado [Nota do Editor]. 
pontos a luz segue o caminho óptico estacionário relativamente aos caminhos ópticos vizinhos que possuem as mesmas extremidades".

Se o meio possui um índice de refração cuja variação é muito rápida, ou então se surgem na propagação da luz obstáculos provocando o aparecimento de fenômenos de interferência ou de difração, o principio de Fermat, que caracteriza a Óptica geométrica, não mais se aplica e torna-se necessário recorrer aos métodos mais rigorosos da Óptica ondulatória.

\section{Equação da propagação das on- das. Ondas luminosas. Óptica geométrica e o seu campo de aplicação. Princípio de Fermat.}

Consideremos um fenômeno físico, representado pela função $\psi(\mathbf{r}, t)$ realizando-se, por hipótese, em um meio não homogêneo, mas permanente e isótropo, caracterizado pela função escalar de ponto $n(\mathbf{r})$. Dizemos que o fenômeno se propaga por ondas se a função $\psi(\mathbf{r}, t)$ satisfaz a equação

$$
\Delta \psi=\frac{n^{2}}{c^{2}} \frac{\partial^{2} \psi}{\partial t^{2}}
$$

Nesta equação $c$ designa uma constante e $\Delta$ o operador "laplaciano"; $\Delta \psi=\operatorname{div} \operatorname{grad} \psi$.

Se o fenômeno físico considerado é um fenômeno luminoso, $\psi(\mathbf{r}, t)$ representa uma "variável luminosa", $n(\mathbf{r})$ é o índice de refração do meio, e $c$ é a velocidade da luz no vácuo. Tomemos um sistema de coordenadas curvilíneas $x_{i}$ $(i=1,2,3)$ cujo elemento de arco $d s$ é dado pela fórmula

$$
d s^{2}=\sum_{i j} a_{i j} d x_{i} d x_{j}
$$

obtemos

$$
\Delta \psi=\frac{1}{\sqrt{a}} \sum_{i} \frac{\partial}{\partial x_{i}}\left(\sqrt{a} \sum_{i} a^{i j} \frac{\partial \psi}{\partial x^{j}}\right)
$$

$a$ designando o determinante dos $a_{i j}$ (sempre positivo); e $a^{i j} . a=A^{i j}$, sendo $A^{i j}$ o complemento algébrico do elemento $a_{i j}$ no determinante $a$. Se as coordenadas $x_{i}$ forem cartesianas e ortogonais, $\Delta \psi=\sum_{i} \frac{\partial^{2} \psi}{\partial x_{i}^{2}}$.

A equação das ondas é, pois, uma equação de derivadas parciais linear, de $2 \mathrm{a}$. ordem, homogênea relativamente às derivadas de $\psi$. A esta equação se aplica o "principio da superposição linear das soluções". Assim se tivermos um numero finito $m$ de soluções $\psi_{k}(\mathbf{r}, t)(k=1,2, \ldots m)$ e $m$ constantes arbitrárias $c_{k}$, a função

$$
\psi(\mathbf{r}, t)=\sum_{k=1}^{k=m} c_{k} \psi_{k}(\mathbf{r}, t)
$$

é uma solução da equação das ondas. Se o número de soluções particulares $\psi_{k}(\mathbf{r}, t)$ for infinito, a função

$$
\psi(\mathbf{r}, t)=\sum_{k=1}^{k=\infty} c_{k} \psi_{k}(\mathbf{r}, t)
$$

será uma solução da equação das ondas caso a série seja convergente e derivável duas vezes termo a termo.

Indiquemos ainda outros tipos de soluções da equação das ondas. Seja $\psi_{1}(\mathbf{r}, t, \alpha)$ uma solução dependendo do parâmetro $\alpha$, a fórmula

$$
\psi(\mathbf{r}, t)=\int_{\alpha_{1}}^{\alpha_{2}} \psi_{1}(\mathbf{r}, t, \alpha) y(\alpha) d \alpha
$$

$y(\alpha)$ designando uma função arbitrária de $\alpha$, também representará uma solução da equação das ondas, caso a integral tenha um sentido e se lhe possa aplicar duas vezes a derivação sob o sinal de integração. Em vez de uma integral simples poderíamos considerar integrais múltiplas soluções da equação das ondas; assim, por exemplo

$$
\psi(\mathbf{r}, t)=\int_{\alpha_{1}}^{\alpha_{2}} \int_{\beta_{1}}^{\beta_{2}} \int_{\gamma_{1}}^{\gamma_{2}} \psi_{1}(\mathbf{r}, t, \alpha, \beta, \gamma) y(\alpha, \beta, \gamma) d \alpha d \beta d \gamma
$$

É fácil obter soluções particulares da equação das ondas. Assim, por exemplo,

$$
\psi(\mathbf{r}, t)=\left(\mathbf{r}-\mathbf{r}_{0}\right) \cdot \mathbf{e}+c_{1} t+c_{2}
$$

é uma solução, quaisquer que sejam as constantes escalares $c_{1}$ e $c_{2}$ e o vetor constante e (em coordenadas cartesianas retangulares $x_{i}, \psi(\mathbf{r}, t)$ seria uma função linear de $x_{i} \mathrm{e}$ t). O que importa, porém, é achar soluções que também satisfaçam às condições impostas pela natureza física da questão.

Nas aplicações aos fenômenos ondulatórios é importante o estudo das soluções particulares do tipo

$$
\psi(\mathbf{r}, t)=a(\mathbf{r}) f[\nu t-\varphi(f r)]
$$

$\nu$ designando uma constante positiva. A função $\Phi(\mathbf{r}, t)=$ $\nu t-\varphi(\mathbf{r})$ recebe a denominação de "fase". As superfícies 
de igual fase $\Phi_{0}$ são representadas por

$$
\Phi=\nu t-\varphi(\mathbf{r})=\Phi_{0}
$$

$\mathrm{ou}$

$$
\varphi(\mathbf{r})=\nu t-\Phi_{0}
$$

Em um dado instante $t_{0}$ a superfície de fase $\Phi_{0}$ coincide com a superfície

$$
\varphi(\mathbf{r})=\nu t_{0}-\Phi_{0}
$$

da família

$$
\varphi(\mathbf{r})=\text { const. }
$$

O tempo crescendo, a superfície de fase $\Phi_{0}$ transportase de uma para outra superfície da família $\varphi(\mathbf{r})=$ const. As linhas ortogonais às superfícies $\varphi(\mathbf{r})=$ const. recebem a denominação de "raios".

Procuremos a velocidade escalar $u$ de fase que, por definição, é a velocidade escalar com a qual se deve deslocar ao longo do raio para acompanhar um valor constante da fase.

Consideremos, no instante $t$ um ponto $P$ (dado por $\mathbf{r}$ ) da superfície

$$
\varphi(\mathbf{r})=\nu t-\Phi
$$

seja, no tempo $d t, d \mathbf{r}$ o deslocamento elementar de $P$ ao longo do raio que passa por $P$, o valor de $\Phi$ permanecendo constante; temos

$$
u=\left|\frac{d \mathbf{r}}{d t}\right| .
$$

Ora, se, para o deslocamento $d \mathbf{r}$, a fase não variou,

$$
d \Phi=\nu d t-\operatorname{grad} \varphi \cdot d \mathbf{r}=0
$$

$\mathrm{ou}$

$$
u=\frac{\nu}{|\operatorname{grad} \varphi|}
$$

Suponhamos agora que a função $f(\Phi)$ seja periódica e de período $\delta$ : $f(\Phi+\delta)=f(\Phi)$. Neste caso $\lambda=\delta /|\operatorname{grad} \varphi|$ recebe a denominação de comprimento de onda. Eis como se justifica esta denominação no caso em que as superfícies da família $\varphi(\mathbf{r})=$ const. são planos paralelos sobre o raio retilíneo, tal que a fase $\Phi$ varie de $\delta$, e tal portanto que $f(\Phi)$ retome seu valor (o tempo permanecendo constante):

$$
\begin{gathered}
\delta=\left|\Delta_{n} \varphi\right|=\left|\operatorname{grad} \varphi \cdot \Delta_{n} \mathbf{r}\right|, \\
\lambda=\left|\Delta_{n} \mathbf{r}\right|=\frac{\delta}{|\operatorname{grad} \varphi|} ;
\end{gathered}
$$

neste caso $\lambda$ é constante. Quando as superficies $\varphi(\mathbf{r})=$ const. não são planas, define-se ainda o comprimento de onda $\lambda$ pela mesma fórmula; $\lambda$ é então função de ponto $\lambda(\mathbf{r})$.

No estudo dos fenômenos luminosos impõe-se a consideração da função periódica

$$
f(\Phi)=e^{2 \pi i \Phi}
$$

donde, para a função de ondas,

$$
\psi(\mathbf{r}, t)=a(\mathbf{r}) e^{2 \pi i[\nu t-\varphi(\mathbf{r})]},
$$

neste caso $\delta=1$ e $u=\lambda \nu ; \nu$ é a freqüência da onda que então se diz monocromática.

Vejamos como determinar a fase $\Phi$ e a amplitude $a(\mathbf{r})$. Observemos preliminarmente que

$$
\frac{\partial^{2} \psi}{\partial t^{2}}=-4 \pi^{2} \nu^{2} \psi
$$

e a equação das ondas torna-se

$$
\Delta \psi+\frac{4 \pi^{2} \nu^{2} n^{2}}{c^{2}} \psi=0
$$

Temos

$$
\Delta \psi=\left[\Delta a-4 \pi^{2} a|\operatorname{grad} \varphi|^{2}-2 \pi i(a \Delta \varphi+2 \operatorname{grad} a \cdot \operatorname{grad} \varphi)\right] e^{2 \pi i \Phi},
$$

e, portanto, a equação

$$
1-\frac{u^{2} n^{2}}{c^{2}} \frac{\Delta a}{a}+i \frac{\lambda^{2}}{2 \pi}\left(\Delta \varphi+\frac{2}{a} \operatorname{grad} a \cdot \operatorname{grad} \varphi\right)=0 .
$$

Igualando a zero separadamente a parte real e a parte imaginária, obtemos as duas equações

$$
\begin{aligned}
& \frac{n^{2} u^{2}}{c^{2}}+\frac{\lambda^{2}}{4 \pi^{2}} \frac{\Delta a}{a}=1 ; \\
& \frac{2}{a} \operatorname{grad} a \cdot \operatorname{grad} \varphi+\Delta \varphi=0 .
\end{aligned}
$$

Na hipótese de um índice de refração constante (meio homogêneo), as duas equações acima podem ser satisfeitas identicamente tomando-se a amplitude $a(\mathbf{r})$ constante, e $\varphi(\mathbf{r})=\left(\mathbf{r}-\mathbf{r}_{0}\right) \cdot \mathbf{e}$, sendo $|\mathbf{e}|=n \nu / c ;$ a equação $\varphi(\mathbf{r})=$ const. representa então uma família de planos paralelos. Este é o caso mais simples da Óptica geométrica.

Se o índice de refração for lentamente variável diremos que as condições da Óptica geométrica são verificadas, se na escala dos comprimentos de onda $\lambda$ as variações da amplitude $a(\mathbf{r})$ e do vetor grad $\varphi$ forem pequenas; neste caso $\lambda^{2}(\Delta a / a)$ é desprezível em face da unidade e obtemos

$$
u=\frac{c}{n(\mathbf{r})}
$$


ou, para determinar $\varphi(\mathbf{r})$, a equação

$$
|\operatorname{grad} \varphi|^{2}=\frac{n^{2} \nu^{2}}{c^{2}}
$$

Seja $\widehat{\mathbf{s}}$ um vetor unitário, normal à superfície $\varphi(\mathbf{r})=$ const., e orientado no sentido dos $\varphi$ crescentes; temos

$$
\operatorname{grad} \varphi=\frac{n \nu}{c} \widehat{\mathbf{s}}
$$

e a fórmula

$$
\operatorname{grad}(\log a) \cdot \widehat{\mathbf{s}}=-\frac{1}{2 n} \operatorname{div}(n \widehat{\mathbf{s}})
$$

para determinar a amplitude $a(\mathbf{r})$.

Estas fórmulas mostram que se a variação do índice de refração for muito rápida, a amplitude $a(\mathbf{r})$ e $\mathrm{o} \operatorname{grad} \varphi$ poderão variar consideravelmente, e a aproximação oferecida pela Óptica geométrica poder-se-á tornar insuficiente. Também nos fenômenos de difração, nos quais há variações bruscas da amplitude, ter-se-á de rejeitar a simplificação trazida pela Óptica geométrica, e de recorrer diretamente à equação das ondas.

Observe-se ainda que a transição da Óptica ondulatória ou Óptica física para a Óptica geométrica poder-se-ia efetuar, considerando-se em cada ponto o comprimento de onda $\lambda$ como infinitamente pequeno e supondo que as outras grandezas na equação se conservassem finitas e contínuas; resultaria, no limite, a equação já achada

$$
|\operatorname{grad} \varphi|^{2}=\frac{n^{2} \nu^{2}}{c^{2}}
$$

ou, em coordenadas curvilíneas $x_{i}$,

$$
\sum_{i j} a^{i j} \frac{\partial \varphi}{\partial x_{i}} \frac{\partial \varphi}{\partial x_{j}}=\frac{n^{2} \nu^{2}}{c^{2}}
$$

equação de derivadas parciais de 1a. ordem e do 2o. grau, e do tipo Hamilton-Jacobi.

Seja $\varphi\left(\mathbf{r}, \alpha_{1}, \alpha_{2}\right)$ uma integral completa desta equação de derivadas parciais, contendo duas constantes arbitrárias $\alpha_{1}$ e $\alpha_{2}$ não aditivas. As equações de uma trajetória ortogonal das superfícies $\varphi=$ const. ou de um "raio" percorrido pela luz, são

$$
\frac{\partial \varphi}{\partial \alpha_{1}}=\beta_{1} \quad \frac{\partial \varphi}{\partial \alpha_{2}}=\beta_{2},
$$

$\beta_{1}$ e $\beta_{2}$ designando duas constantes.

Com efeito, se $d \mathbf{r}$ é um deslocamento elementar ao longo da linha representada pelas 2 equações acima:

$$
\operatorname{grad}\left(\frac{\partial \varphi}{\partial \alpha_{1}}\right) \cdot d \mathbf{r}=0, \quad \operatorname{grad}\left(\frac{\partial \varphi}{\partial \alpha_{2}}\right) \cdot d \mathbf{r}=0 .
$$

Por outro lado derivando $|\operatorname{grad} \varphi|^{2}=n^{2} \nu^{2} / c^{2}$ relativamente a $\alpha_{1}$ e a $\alpha_{2}$ :

$$
\operatorname{grad} \varphi \cdot \frac{\boldsymbol{\partial}(\operatorname{grad} \varphi)}{\boldsymbol{\partial} \alpha_{1}}=0, \quad \operatorname{grad} \varphi \cdot \frac{\boldsymbol{\partial}(\operatorname{grad} \varphi)}{\boldsymbol{\partial} \alpha_{21}}=0
$$

ora

$$
\frac{\partial(\operatorname{grad} \varphi)}{\partial \alpha_{1}}=\operatorname{grad}\left(\frac{\partial \varphi}{\partial \alpha_{1}}\right)
$$

vemos, pois, que $d \mathbf{r}$ e grad $\varphi$ são paralelos, e a linha considerada é uma trajetória ortogonal das superfícies $\varphi(\mathbf{r})=$ const.. A fórmula

$$
d \varphi=\operatorname{grad} \varphi \cdot \mathbf{r}=\frac{n \nu}{c} d s
$$

conduz a

$$
\varphi=\frac{\nu}{c} \int n(\mathbf{r}) d s,
$$

a integral, tomada ao longo de um "raio" isto é ao longo de uma trajetória ortogonal das superfícies $\varphi(\mathbf{r})=$ const., representa o caminho óptico seguido pela luz. Como

$$
u=\frac{c}{n}=\frac{d s}{d t}
$$

tem-se, também,

$$
\varphi=\nu \int d t
$$

$\varphi$ é pois proporcional ao tempo gasto pela luz para percorrer, entre 2 pontos, um determinado "raio". Pelo princípio de Fermat do qual decorrem, como sabemos as proposições elementares fundamentais da Óptica geométrica tais como as leis da refração de Descartes, o caminho óptico seguido pela luz, entre dois pontos, é estacionário, e se deve ter

$$
\delta \int n(\mathbf{r}) d s=0
$$

Conforme ver-se-á mais adiante, o princípio de Fermat conduz efetivamente à equação de derivadas parciais da Óptica geométrica e permite a determinação dos "raios" percorridos pela luz.

\section{O princípio de Hamilton na Mecânica clássica. Equações canônicas. Equação de Hamilton- Jacobi. Analogia entre a Mecânica clássica e a Óptica geométrica}

O princípio de Fermat sugeriu a Hamilton a descoberta do célebre princípio que recebeu seu nome e do qual decorrem, sob certas condições, as equações do movimento dos sistemas da Mecânica clássica.

Vamos enunciar o princípio de Hamilton para o caso de uma partícula de massa $m$ cuja posição é determinada no espaço por um sistema de coordenadas curvilíneas $x_{i}$. A força viva [energia cinética] $T$ da partícula é dada pela fórmula

$$
T=\frac{1}{2} m v^{2}=\frac{1}{2} m\left(\frac{d \mathbf{r}}{d t}\right)^{2} .
$$


Temos

$$
|d \mathbf{r}|^{2}=d s^{2}=\sum_{i j} a_{i j} d x_{i} d x_{j}
$$

pondo $\dot{x}_{k}=\left(d x_{k} / d t\right)$ vem

$$
T=\frac{m}{2} \sum_{i j} a_{i j} \dot{x}_{i} \dot{x}_{j}
$$

Seja $U(\mathbf{r}, t)$ ou $U\left(x_{i}, t\right)$ a função potencial que representa a influência das ações exteriores, no caso de um campo não permanente.

A função

$$
\mathcal{L}\left(x_{i}, \dot{x}_{i}, t\right)=T\left(x_{i}, \dot{x}_{i}\right)-U\left(x_{i}, t\right)
$$

recebe a denominação de função de Lagrange e tem as dimensões de uma energia.

A integral $V=\int_{t_{0}}^{t} \mathcal{L} d t$ é denominada integral de Hamilton e tem as dimensões do produto de uma energia por um tempo, isto é as dimensões de uma "ação".

Sejam $P_{0}$ e $P$ as posições do móvel nos tempos $t_{0}$ e $t$, respectivamente. O principio de Hamilton afirma que: "entre as trajetórias infinitamente vizinhas que levam o móvel de $P_{0}$ a $P$ no mesmo intervalo de tempo, a trajetória real é aquela que torna estacionária a integral $V$ de Hamilton; deve-se ter, pois, para a trajetória realmente percorrida pelo móvel

$$
\delta \int_{t_{0}}^{t} \mathcal{L} d t=0
$$

quando se faz variar infinitamente pouco a forma das funções $x_{i}(t)$ e por conseguinte de $\dot{x}_{i}(t)$, os valores iniciais e finais dos variáveis $x_{i}$ permanecendo fixos.

Sabemos, pelo Cálculo das Variações, que se $\int_{t_{0}}^{t} \mathcal{L} d t$ é estacionária, as 3 derivadas variacionais de $\mathcal{L}$ relativamente aos $x_{i}$ são nulas

$$
\frac{\partial \mathcal{L}}{\partial x_{i}}-\frac{d}{d t}\left(\frac{\partial \mathcal{L}}{\partial \dot{x}_{i}}\right)=0 ; \quad(i=1,2,3)
$$

são as equações de $2 \mathrm{a}$. ordem, de Lagrange, que permitem determinar o movimento da partícula em função de 6 constantes arbitrárias, p. ex. as coordenadas iniciais $\left(x_{i}\right)_{0}$ do ponto $P$ e os valores iniciais $\left(\dot{x}_{i}\right)_{0}$ da velocidade.

O sistema de 3 equações de $2 \mathrm{a}$. ordem de Lagrange pode ser substituido por um sistema de equações de $1 \mathrm{a}$. ordem de Hamilton, introduzindo as novas variáveis $p_{i}=\boldsymbol{\partial \mathcal { L }} / \boldsymbol{\partial} \dot{x}_{i}$ que recebem a denominação de momentos conjugados das variáveis $x_{i}$. Consideremos para isso a função de Hamilton

$$
\mathcal{H}\left(x_{i}, p_{i}, t\right)=\sum p_{i} \dot{x}_{i}-\mathcal{L}\left(x_{i}, \dot{x}_{i}, t\right)
$$

supomos, no 2o. membro, os $\dot{x}_{i}$ expressos em função dos $x_{i}, p_{i}$, e $t$ por intermédio das fórmulas de transformação.

Como $T$ é homogênea relativamente aos $\dot{x}_{i}$ :

$$
2 T=\sum \dot{x}_{i} \frac{\partial \mathcal{L}}{\partial \dot{x}_{i}}=\sum p_{i} \dot{x}_{i}
$$

e

$$
\mathcal{H}=2 T-(T-U)=T+U
$$

Derivemos $\mathcal{H}$ relativamente a $x_{i}$ e $p_{i}$ :

$$
\frac{\partial \mathcal{H}}{\partial x_{i}}=-\frac{\partial \mathcal{L}}{\partial x_{i}}=-\frac{d p_{i}}{d t}, \quad \frac{\partial \mathcal{H}}{\partial p_{i}}=\dot{x}_{i}=\frac{d x_{i}}{d t} ;
$$

é o sistema das equações canônicas de Hamilton. Calcule$\operatorname{mos} d \mathcal{H} / d t$; obtemos

$$
\frac{d \mathcal{H}}{d t}=\frac{\partial \mathcal{H}}{\partial t} .
$$

Se o campo é permanente, $\mathcal{H}$ não depende do tempo, e $\mathcal{H}$ = const $=E$, ou $T+U=E$, é o teorema da conservação da energia total. No caso do campo permanente pode-se enunciar o princípio de Hamilton sob a forma conhecida pelo nome de "princípio da menor ação". Temos:

$$
V=\int_{t_{0}}^{t} \mathcal{L} d t=\int_{t_{0}}^{t}(2 T-E) d t=S-E\left(t-t_{0}\right)
$$

pondo

$$
S=\int_{t_{0}}^{t} 2 T d t
$$

$\mathrm{Se}$

$$
\delta \int_{t_{0}}^{t} \mathcal{L} d t=0, \quad \text { vem } \quad \delta \int_{t_{0}}^{t} 2 T d t=0 ;
$$

$S$ recebe o nome de "ação". Observemos que

$$
\begin{gathered}
2 T=m \frac{d s^{2}}{d t^{2}} \quad \text { e } 2 T d t=\sqrt{2 T} \sqrt{m} d s, \\
S=\int_{P_{0}}^{P} \sqrt{2 m(E-U)} d s,
\end{gathered}
$$

a integral sendo tomada ao longo da trajetória da partícula.

Conhecida a constante $E$ e dada a posição inicial do móvel, a velocidade inicial deste fica determinada em grandeza mas não em direção. Pode-se enunciar o princípio da menor ação: "Sejam $P_{0}$ e $P$ dois pontos fixos. Lançandose de $P_{0}$ móvel em uma direção tal que ele atinja o ponto $P$, a trajetória por ele descrita goza da propriedade de tornar nula a variação da ação $S$ quando se passa da trajetória real para uma trajetória infinitamente vizinha que possui as mesmas extremidades."

Com o objetivo de pôr em evidência a analogia entre a Mecânica clássica e a Óptica geométrica, vamos mostrar: 1) a "ação" $S=\int_{t_{0}}^{t} 2 T d t$ satisfaz a uma equação de derivadas parciais de 1a. ordem do tipo Hamilton-Jacobi, análoga à equação da Óptica geométrica; 2) as trajetórias do ponto móvel são ortogonais às superfícies $S=$ const., assim como os raios luminosos são ortogonais às superfícies $\varphi=$ const.

Admitiremos que, nas demonstrações, as funções sejam univalentes, contínuas e deriváveis.

1) Temos, pondo $\mathbf{p}=m \mathbf{v}$

$$
\begin{aligned}
& S=\int_{t_{0}}^{t} 2 T d t=\int_{t_{0}}^{t} \mathbf{p} \cdot \frac{d \mathbf{r}}{d t} d t \\
& d S=(\operatorname{grad} S) \cdot d \mathbf{r}=\mathbf{p} \cdot d \mathbf{r}
\end{aligned}
$$


resulta

$$
\begin{aligned}
& \operatorname{grad} S=\mathbf{p} \\
& \operatorname{grad} S^{2}=m v^{2}=2 m(E-U) .
\end{aligned}
$$

$S$ satisfaz, pois, à equação de derivadas parciais de Hamilton-Jacobi

$$
\sum_{i j} a^{i j} \frac{\partial S}{\partial x_{i}} \frac{\partial S}{\partial x_{j}}=2 m(E-U)
$$

análoga à equação da Óptica geométrica.

2) Como

$$
\operatorname{grad} S=\mathbf{p}=\mathbf{m} \frac{d \mathbf{r}}{d t},
$$

a tangente à trajetória do ponto $P$ é paralela ao vetor grad $S$ e portanto normal à superficie $S=$ const. que passa por $P$.

As equações das trajetórias ortogonais das superficies $S=$ const. e portanto as equações das trajetórias do móvel, são dadas pelas equações $\boldsymbol{\partial} S / \boldsymbol{\partial} \alpha_{1}=$ const., $\boldsymbol{\partial} S / \boldsymbol{\partial} \alpha_{2}=$ const., $S\left(\mathbf{r}, \alpha_{1}, \alpha_{2}\right)$ designando uma integral completa da equação de Hamilton-Jacobi, contendo 2 constantes arbitrárias não aditivas $\alpha_{1}$ e $\alpha_{2}$. A demonstração desta proposição se faz como para o caso dos raios luminosos quando consideramos a equação da Óptica geométrica.

A analogia entre a Mecânica clássica e a Óptica geométrica ainda mais se evidencia traduzindo as fórmulas da Mecânica em linguagem das ondas e comparando os resultados com os que anteriormente foram obtidos para a Óptica.

Seja $V(\mathbf{r}, \mathbf{t})=S(\mathbf{r})-E t$ a função de Hamilton na qual se tomou $t_{0}$ como origem dos tempos. Consideremos, num dado instante, a família de superfícies $V=S(\mathbf{r})-E t=$ const., grad $V$ ou grad $S$ é um vetor normal à superfície, em cujos pontos $V$ é constante e também é, como vimos, tangente à trajetória da partícula de massa $m$.

Tomemos uma superficie $V=$ const. correspondente ao valor $V_{0}$ de $V$; crescendo o tempo a superfície $V$ transportase de uma para outra superfície da familia $S(\mathbf{r})=$ const. Calculemos, nesse movimento, a velocidade escalar $u$ de um ponto $P$ da superfície $V_{0}$, contada normalmente à mesma superfície. Temos, se $V$ conserva o valor constante $V_{0}$ :

$$
d V=0=\operatorname{grad} V \cdot d \mathbf{r}+\frac{\partial V}{\partial t} d t
$$

donde

$$
u=\left|\frac{d \mathbf{r}}{d t}\right|=\frac{-(\boldsymbol{\partial} V / \boldsymbol{\partial} t)}{|\operatorname{grad} V|}=\frac{E}{\sqrt{2 m(E-U)}}
$$

pois grad $V^{2}=|\operatorname{grad} S|^{2}=2 m(E-U)$.

Consideremos agora um meio permanente e isótropo cujo índice de refração é $n(\mathbf{r})$. Suponhamos que as condições de aplicação da Óptica geométrica sejam verificadas e tomemos $-V / h=E / h-S(\mathbf{r}) / h$ como a fase de uma onda luminosa; $h$ é uma constante com as dimensões de uma ação, e $V / h$ é portanto um número puro, como convém à fase. A freqüência $\nu$ da onda deve ser tomada igual a $E / h$, e a velocidade de fase torna-se

$$
u=\frac{h \nu}{|\operatorname{grad} S|}=\frac{E}{m v}=\frac{E}{\sqrt{2 m(E-U)}} .
$$

Tem-se para o índice de refração $n(\mathbf{r})$

$$
n(\mathbf{r})=\frac{c}{u}=\frac{c \sqrt{2 m(E-U)}}{E} .
$$

A equação de Hamilton-Jacobi toma então o aspecto

$$
\left|\operatorname{grad} \frac{S}{h}\right|^{2}=\frac{2 m(E-U)}{h^{2}}=\frac{n^{2} \nu^{2}}{c^{2}} .
$$

Fazemos corresponder a uma partícula de massa $m$ em movimento, uma onda monocromática de frequiência a qual é proporcional à energia $E$ da partícula. $\mathrm{O}$ comprimento de onda $\lambda$ proporcional à constante $h: \lambda=u / \nu=h / m v$; esta é a fórmula que De Broglie obteve recorrendo à teoria da relatividade.

Ao princípio da menor ação no movimento do ponto $P$ de massa $m$, corresponde o princípio de Fermat. Com efeito

$$
S=\int_{P_{0}}^{P} \sqrt{2 m(E-U)} d s=\frac{E}{c} \int_{P_{0}}^{P} n(\mathbf{r}) d s .
$$

Se a ação $S$ é estacionária para a trajetória real descrita pelo móvel, vem $\delta \int n(\mathbf{r}) d s=0$ que é a expressão analítica do Princípio de Fermat: "ao longo de uma trajetória ortogonal das superfícies de igual fase o caminho óptico é estacionário relativamente aos caminhos ópticos infinitamente vizinhos que possuem as mesmas extremidades."

A analogia entre a Mecânica clássica e a Óptica geométrica é completa quando se compara a trajetória de um ponto móvel com um raio luminoso.

Já vimos que as leis da Óptica geométrica constituem uma aproximação das leis da Óptica ondulatória ou Óptica física, quando o comprimento de onda $\lambda$ é pequeno relativamente às dimensões das outras grandezas que intervêm nos fenômenos considerados. Salientamos que para os fenômenos de interferência e de difração essa condição não é geralmente satisfeita e a aproximação fornecida pela Óptica geométrica se torna insuficiente.

O princípio de Hamilton, na Mecânica clássica, aplicase corretamente ao estudo do movimento dos sistemas macroscópicos, quando aplicado aos fenômenos atômicos conduz, como é notorio, a resultados pouco satisfatórios.

Schrödinger salienta a analogia entre as condições que provocam o fracasso do Princípio de Fermat no estudo de certos fenômenos ópticos, e as que determinam o fracasso do princípio de Hamilton quando aplicado aos fenômenos atômicos, onde é fundamental, conforme veremos, a consideração da relação de Planck $E=h \nu(h=$ $6.55 \times 10^{-17}$ C. G. S. designando a célebre constante de Planck). Para Schrödinger ambos os fracassos são devidos à mesma causa: as dimensões das grandezas que intervêm nos fenômenos considerados são pequenas relativamente ao comprimento de onda $\lambda$. Em um fenômeno atômico, isto 
corresponde a dizer que, nas equações, a constante de Planck $h$, a que é proporcional $\lambda$ não pode ser desprezada em face das outras grandezas.

Schrödinger procura, então, elaborar para o estudo dos fenômenos atômicos, uma Mecânica ondulatória que conserve para com a Mecânica clássica a mesma relação que a Óptica ondulatória guarda para com a Óptica geométrica.

Continua

\section{Sobre o autor}

Theodoro Augusto Ramos (1895-1936), paulista da Capital, graduou-se pela Escola Politécnica do Rio de Janeiro em engenharia civil e obteve no ano seguinte o grau de doutor em Ciências Físicas e Matemáticas com a tese "Sobre as Funções de Variáveis Reais". Segundo Oliveira Castro ${ }^{1}$, a tese "representa certamente a contribuição mais importante que pode apresentar a pesquisa matemática brasileira, antes da criação da FFCL da USP.” No mesmo ano conseguiu uma posição acadêmica na Escola Politécnica de São Paulo assumindo, em 1926, a cátedra de "Vetores, Geometria Analítica, Geometria Projetiva e Aplicação à Nomografia”. Em 1923, apresentou na Academia Brasileira de Ciências (ABC) o primeiro trabalho original sobre a teoria quântica no Brasil "A Theoria da Relatividade e as Raias Espectrais do Hydrogenio" [publicado na Revista Polytechnica, n. 74, pp. 181-188, set./dez. (1923)], e que apareceu nos anais da ABC apenas em 1929 [v. 1, n. 1, pp. 20-27]. Publicou vários trabalhos originais em matemática, o que era incomum na época. Foi membro de várias comissões de ensino de engenharia, inclusive membro do Conselho Nacional de Educação. Atuou ainda com eficiência no governo paulista na área de saneamento básico. Foi Secretário de Educação e Saúde Pública e prefeito de São Paulo pelo período de três meses em 1933. Em 1934, participou da missão à Europa para contratar professores para a recém criada Faculdade de Filosofia, Ciências e Letras (FFCL) da USP da qual foi Diretor. [N.E] 